\title{
In utero exposure to mercury and childhood overweight or obesity: counteracting effect of maternal folate status
}

Guoying Wang $^{1 *+}$, Jessica DiBari ${ }^{2+}$, Eric Bind ${ }^{3}$, Andrew M. Steffens ${ }^{3}$, Jhindan Mukherjee ${ }^{3}$, Tami R. Bartell ${ }^{4}$, David C. Bellinger ${ }^{5}$, Xiumei Hong ${ }^{1}$, Yuelong $\mathrm{Ji}^{1}$, Mei-Cheng Wang ${ }^{6}$, Marsha Wills-Karp ${ }^{7}$, Tina L. Cheng ${ }^{8}$ and Xiaobin Wang ${ }^{1,8}$

\begin{abstract}
Background: Low-dose mercury $(\mathrm{Hg})$ exposure has been associated with cardiovascular diseases, diabetes, and obesity in adults, but it is unknown the metabolic consequence of in utero $\mathrm{Hg}$ exposure. This study aimed to investigate the association between in utero $\mathrm{Hg}$ exposure and child overweight or obesity (OWO) and to explore if adequate maternal folate can mitigate $\mathrm{Hg}$ toxicity.

Methods: This prospective study included 1442 mother-child pairs recruited at birth and followed up to age 15 years. Maternal $\mathrm{Hg}$ in red blood cells and plasma folate levels were measured in samples collected 1-3 days after delivery (a proxy for third trimester exposure). Adequate folate was defined as plasma folate $\geq 20.4 \mathrm{nmol} / \mathrm{L}$. Childhood OWO was defined as body mass index $\geq 85 \%$ percentile for age and sex.
\end{abstract}

Results: The median (interquartile range) of maternal Hg levels were 2.11 (1.04-3.70) $\mu \mathrm{g} / \mathrm{L}$. Geometric mean (95\% Cl) of maternal folate levels were 31.1 (30.1-32.1) nmol/L. Maternal Hg levels were positively associated with child OWO from age 2-15 years, independent of maternal pre-pregnancy OWO, diabetes, and other covariates. The relative risk $(\mathrm{RR}=$ $1.24,95 \% \mathrm{Cl} 1.05-1.47$ ) of child OWO associated with the highest quartile of $\mathrm{Hg}$ exposure was $24 \%$ higher than those with the lowest quartile. Maternal pre-pregnancy OWO and/or diabetes additively enhanced $\mathrm{Hg}$ toxicity. The highest risk of child OWO was found among children of OWO and diabetic mothers in the top $\mathrm{Hg}$ quartile (RR $=2.06 ; 95 \% \mathrm{Cl}$ 1.56-2.71) compared to their counterparts. Furthermore, adequate maternal folate status mitigated $\mathrm{Hg}$ toxicity. Given top quartile $\mathrm{Hg}$ exposure, adequate maternal folate was associated with a $34 \%$ reduction in child OWO risk (RR $=0.66$, $95 \% \mathrm{Cl} 0.51-0.85)$ as compared with insufficient maternal folate. There was a suggestive interaction between maternal $\mathrm{Hg}$ and folate levels on child OWO risk ( $p$ for interaction $=0.086$ ).

Conclusions: In this US urban, multi-ethnic population, elevated in utero Hg exposure was associated with a higher risk of OWO in childhood, and such risk was enhanced by maternal OWO and/or diabetes and reduced by adequate maternal folate. These findings underscore the need to screen for $\mathrm{Hg}$ and to optimize maternal folate status, especially among mothers with OWO and/or diabetes.

Keywords: Diabetes, Folate, In utero, Mercury, Metal, Nutrient, Overweight, Obesity

\footnotetext{
* Correspondence: gwang24@jhu.edu

${ }^{+}$Guoying Wang and Jessica DiBari Co-first authors: these authors made equal contributions.

${ }^{1}$ Department of Population, Family and Reproductive Health, Center on the

Early Life Origins of Disease, Johns Hopkins Bloomberg School of Public

Health, 615 N. Wolfe Street, Baltimore, MD 21205-2179, USA

Full list of author information is available at the end of the article
}

(c) The Author(s). 2019 Open Access This article is distributed under the terms of the Creative Commons Attribution 4.0 International License (http://creativecommons.org/licenses/by/4.0/), which permits unrestricted use, distribution, and reproduction in any medium, provided you give appropriate credit to the original author(s) and the source, provide a link to the Creative Commons license, and indicate if changes were made. The Creative Commons Public Domain Dedication waiver (http://creativecommons.org/publicdomain/zero/1.0/) applies to the data made available in this article, unless otherwise stated. 


\section{Background}

Mercury $(\mathrm{Hg})$ is a persistent and widespread environmental pollutant and is highly toxic to human health worldwide. Exposure to toxic $\mathrm{Hg}$ is ubiquitous in the general US population [1]. The National Health and Nutrition Examination Survey found that $85.2 \%$ of the population had detectable levels of $\mathrm{Hg}$ [1]. Methylmercury $(\mathrm{MeHg}$, the major constituent of organic $\mathrm{Hg}$ ) is of particular concern due to its ability to cross the placenta and blood-brain barrier during pregnancy, as well as bioaccumulation [2]. Neuro-toxic effects of $\mathrm{MeHg}$ have been well studied [3], and MeHg's role in cardiometabolic health is beginning to be recognized $[4,5]$. Studies in adults have found that low-dose $\mathrm{Hg}$ exposure was associated with obesity [6] and visceral adipose tissue [7], suggesting that $\mathrm{Hg}$ is an obesogen.

The developing fetus is particularly vulnerable to nutritional and environmental exposures [8, 9]. Previous studies suggest that obesity epidemic could be, in part, due to in utero exposures to adverse environments $[10,11]$. Although $\mathrm{Hg}$ has been recognized as an obesogen $[6,12,13]$, literature is limited for the link of in utero $\mathrm{Hg}$ exposure and obesity in later life. Especially, the role of $\mathrm{Hg}$ in the inter-generational risk of obesity is yet to be explored. Given childhood obesity persists into adulthood and is linked to cardiovascular disease in later life, a critical unanswered question is whether in utero low-dose $\mathrm{Hg}$ exposure increases a child's risk for obesity in childhood and beyond.

In light of widespread maternal exposure to $\mathrm{Hg}$ in the USA, there is an urgent need to identify potentially important mitigating factors. Because antioxidants are desirable in the process of $\mathrm{Hg}$ detoxification [14], folate, an essential B vitamin, may be a candidate due to its antioxidant and anti-inflammatory properties $[15,16]$. Studies have demonstrated that folate has protective effects on cardiovascular diseases [17] and might reduce the adverse impacts of environmental chemicals on the fetus [18]. One study has documented an inverse relationship between serum folate levels and blood Hg concentration during pregnancy [19]. Furthermore, our previous studies unveiled a protective effect of folate on intergenerational risks of obesity and elevated blood pressure $[11,20]$. However, the role of folate in the setting of in utero $\mathrm{Hg}$ exposure and inter-generational link of obesity is yet to be examined.

Using a well-established, population-based birth cohort, this study sought to characterize the potential adverse effect of in utero $\mathrm{Hg}$ exposure on childhood overweight or obesity (OWO) up to 15 years of age. We also assessed whether maternal pre-pregnancy OWO and/or diabetes enhances the toxic effect of in utero $\mathrm{Hg}$ exposure. In addition, we evaluated to what extent adequate maternal folate status during pregnancy counteracts the toxicity of in utero $\mathrm{Hg}$ exposure on childhood OWO.

\section{Study population and methods Study population}

The study protocol was approved by the Institutional Review Boards of Boston Medical Center and Johns Hopkins Bloomberg School of Public Health. Written informed consent was obtained from each study child's biological mother or the study child. Participants of this study came from the Boston Birth Cohort (BBC), who were enrolled at birth and followed prospectively thereafter at the Boston Medical Center. Detailed information on participant enrollment has been described previously [21]. Briefly, the mother-infant pairs were enrolled 24 to $72 \mathrm{~h}$ after delivery. After obtaining written informed consent, trained research staff interviewed mothers using a standardized questionnaire. Maternal blood was drawn for measurement of $\mathrm{Hg}$ in red blood cell (RBC) and plasma folate concentrations. Pertinent clinical information was obtained by a review of maternal and infant medical records, including prenatal ultrasonographic reports, laboratory reports, and information on pregnancy complications, and birth outcomes. As of July 2018, 3163 children who enrolled in the $\mathrm{BBC}$ and received primary care at the Boston Medical Center were followed. Of these children, 1551 mothers had measurements taken for $\mathrm{Hg}$ concentration in the RBCs. There were 109 children excluded due to a lack of body mass index (BMI) data between ages 2-15 years. Finally, this study included 1442 mother-child pairs who had complete data (Additional file 1: Figure S1). The characteristics were similar between the included and excluded samples, except that more Black women and less preterm births were in the included samples (Additional file 1: Table S1).

\section{Ascertainment of maternal $\mathrm{Hg}$ levels in $\mathrm{RBCs}$}

Maternal $\mathrm{RBC}-\mathrm{Hg}$ concentrations were measured using inductively coupled plasma mass spectrometry (ICP-MS) on an Agilent 8900 QQQ (Agilent Technologies Inc., Santa Clara, CA) at the New Jersey Department of Health, Environmental and Chemical Laboratory Services, Metals Laboratory, Trenton, New Jersey. This laboratory is certified by the US Centers for Disease Control and Prevention, Clinical Laboratory Improvement Amendment, and Clinical Laboratory Improvement Services for analysis of clinical samples. The analytical procedures followed standard protocols for quality control and assurance. The quality control includes a calibration curve comprised of a minimum of five points, continuous calibration verification, continuous calibration blank verification, duplicate samples, laboratory fortified matrix samples, blind duplicates, and a minimum of four second source quality 
control samples for every run. Calibration stock solution (catalog\# SM-2107-042-50, High-Purity Standards, SC) and second source quality control samples (Blood Metals Quality Control Specimens, Lot 018, Wisconsin State Laboratory of Hygiene, WI) were used as National Institute of Standards and Technology (NIST)-traceable certified reference materials to calibrate and monitor the accuracy and interday and intraday repeatability of the analysis. The second source samples must recover within the established recovery range set by the manufacturer or within a tighter range established by the laboratory. The coefficient of variation $(\mathrm{CV})$ was $<5.0 \%$. The limit of detection (LOD) was $0.28 \mu \mathrm{g} / \mathrm{L}$. If the concentration was below the LOD, the value was assigned to be the value of the LOD divided by the square root of two.

\section{Ascertainment of maternal plasma folate concentrations}

Maternal plasma folate concentrations were measured by a commercial laboratory via chemiluminescent immunoassay using a MAGLUMI 2000 Analyzer (Snibe Co., Ltd., Shenzhen, China). Each run included a calibration curve with five points and three internal quality controls. If the value of folate was above the maximum LOD, plasma samples were diluted at a ratio of 1:2 and then re-run. The inter-assay CV was < $4 \%$ [22]. In our previous study [11], we found that maternal plasma folate level $\geq 20.4 \mathrm{nmol} / \mathrm{L}$ was associated with a lower risk of child OWO and mitigated the inter-generational link of obesity. As such, we defined adequate maternal folate as maternal plasma folate $\geq 20.4 \mathrm{nmol} / \mathrm{L}$.

\section{Definition of maternal characteristics}

Maternal variables, including age at time of delivery, race/ethnicity, parity, education, pre-pregnancy height and weight, cigarette smoking, and fish consumption, were based on maternal questionnaire interview. Maternal pre-pregnancy BMI was calculated as prepregnancy weight $(\mathrm{kg})$ divided by squared height $(\mathrm{m})$, and further dichotomized into non-OWO (BMI $<25 \mathrm{~kg} / \mathrm{m}^{2}$ ) and OWO (BMI $\geq 25 \mathrm{~kg} / \mathrm{m}^{2}$ ). Fish consumption was grouped into none, 1-2 servings/week, and $\geq 3$ servings/week. Maternal pregnancy complications, including diabetes mellitus (either gestational diabetes or pre-existing diabetes), hypertensive disorders (pre-eclampsia, eclampsia, gestational hypertension, chronic hypertension, and HELLP [hemolysis, elevated liver enzymes, and low platelets syndrome]), were abstracted from medical records. Gestational age was estimated based on the first day of the last menstrual period, as recorded in the maternal medical record, or early ( $<20$ weeks) prenatal ultrasonographic results, as detailed in a previous publication [21].

\section{Assessment of child's birth outcomes and breastfeeding status}

Child's birthweight and gender were abstracted from the medical records. Birthweight for gestational age was calculated according to an established local reference population, and controlled for infant gender, gestational age, and ethnicity [21]. Fetal growth pattern was defined by birthweight for gestational age and grouped into small for gestational age (SGA) (gestational age specific birthweight $<10$ th percentile), large for gestational age (LGA) (birthweight $>90$ th percentile), and appropriate for gestational age (AGA) (birthweight in the 10th to 90th percentile for gestational age). Information regarding infant breastfeeding history was primarily assessed within the first 2 years of follow-up visits, and grouped into exclusively breastfeeding, exclusively formula feeding, or mixed breast and formula feeding [23].

\section{Child BMI and overweight or obesity in childhood}

Child weight and height were extracted from the medical records, which were measured by trained medical staff using the same clinical protocol and equipment during well-child visits. Before data analyses, careful data checking and cleaning of weight and height data was performed. At first, we removed extreme or biologically implausible values. Then, we identified outliners or erroneous height and weight values based on growth curve. When possible, erroneous height and weight values were corrected, otherwise the points were deleted. Age- and sex-specific BMI $z$-scores and percentiles were calculated using US national reference data [24], derived from a US representative sample. OWO was defined as BMI $\geq 85$ th percentile for age and sex [25]. The BBC has used rolling enrollment, so the length of postnatal follow-up and number of well-child visits varied greatly across study participants. It is known that OWO at an older age is more likely to persist into adulthood. Thus, we chose the last visit in each time period as the end point for OWO.

\section{Statistical analysis}

Demographic and clinical data are presented as either mean \pm standard deviation (SD) or $n(\%)$ stratified by maternal $\mathrm{Hg}$ categories. Unadjusted trend $p$ values across maternal $\mathrm{Hg}$ categories were calculated by MantelHaenszel $\chi^{2}$ for categorical variables and linear regression for continuous variables. The relationship between maternal $\mathrm{Hg}$ and child OWO is displayed using locally weighted regression smoothing plots (implemented using PROC LOESS in SAS, a nonparametric regression method).

Relative risk (RR) was estimated by Poisson regression with robust error variance to determine an association between maternal $\mathrm{Hg}$ and child OWO, adjusting for 
important covariates, including maternal age, race/ethnicity, education, parity, smoking, pre-pregnancy OWO, diabetes, hypertensive disorders, preterm birth, fetal growth pattern, and child's breastfeeding status. To examine the persistence of the association from preschool age to school age to adolescents, we performed similar analyses stratified by child age at outcome assessment (e.g., at age 2-5 years, 6-9 years, and 10-15 years). In addition, we evaluated the joint risk attributed to maternal $\mathrm{Hg}$ categories and either or both pre-pregnancy OWO and diabetes. We tested the interaction of maternal pre-pregnancy OWO (as a binary variable) and $\mathrm{Hg}$ levels (as a continuous variable) or the interaction of maternal diabetes status and $\mathrm{Hg}$ levels on child's risk of OWO by adding a multiplicative term in the models. Effect modification was assessed by the likelihood ratio test using an a priori $\alpha$ value of 0.05 . We used same methods to investigate whether maternal adequate folate status can ameliorate the adverse effect of maternal $\mathrm{Hg}$ on child risk of OWO.

Finally, to examine the robustness of the results and biological plausibility, we conducted a series of sensitivity analyses: subgroup analyses, including among fish consumers, term births, Black children, and breastfed children, and sequential models adding more covariables of interest. We also performed similar analyses stratified by child's sex. All $p$ values were from two-sided tests, and all statistical analyses were performed using SAS v.9.4 (SAS Institute Inc., Cary, NC, USA).

\section{Results}

Our study population consisted of 1442 mother-child pairs of which $722(50.1 \%)$ children were boys and 967(67.1\%) were Black. The age range of children at his/ her last visit was $2-15$ years. $\mathrm{Hg}$ was detected in $89 \%$ of mothers. The median [interquartile range (IQR)] of maternal RBC-Hg was $2.11(1.04-3.70) \mu \mathrm{g} / \mathrm{L}$. The distribution of maternal $\mathrm{RBC}-\mathrm{Hg}$ is presented according to race/ethnicity and maternal OWO/diabetes status (Additional file 1: Figure S2). Geometric mean [95\% confidence interval (CI)] of maternal folate plasma was $31.1(30.1-32.1) \mathrm{nmol} / \mathrm{L}$. In all, $21.4 \%$ of mothers had plasma folate levels $<20.4 \mathrm{nmol} / \mathrm{L}$. Maternal and child characteristics stratified by maternal $\mathrm{Hg}$ quartiles are presented in Table 1. Older, non-smoking, Black, and multiparous mothers had higher $\mathrm{Hg}$ levels. A higher frequency of fish intake was associated with higher $\mathrm{Hg}$ levels. Lower plasma folate levels were associated with increasing $\mathrm{Hg}$ levels. Children of mothers with higher $\mathrm{Hg}$ levels tended to be female, older, and breastfed.

\section{Maternal RBC-Hg levels and child OWO}

As shown in Fig. 1, maternal $\mathrm{Hg}$ levels were positively associated with an increased risk of child OWO (panel a). When stratified by maternal pre-pregnancy OWO and/or diabetes, the association was strongest among OWO and diabetic mothers (panel b). Furthermore, the risk of child OWO among OWO and diabetic mothers was the highest across the whole $\mathrm{Hg}$ spectrum. Multivariate regression models (Table 2) showed that the increased risk of child OWO was mainly concentrated in the top $\mathrm{Hg}$ quartile (Q4) with an RR of 1.24 (95\% CI 1.05-1.47), while second (Q2) and third (Q3) Hg quartiles were not significantly associated with the risk of child OWO, as compared with the lowest quartile (Q1). When we combined Q1-Q3 as the reference, the top Hg quartile was associated with increased risk of child OWO (RR $=1.19 ; 95 \%$ CI $1.05-1.35 ; p=0.007)$. These associations persisted from preschool age (2-5 years), to school age (6-9 years) and into early adolescence (1015 years).

\section{Combined effects of maternal pre-pregnancy OWO/ diabetes and $\mathrm{Hg}$ levels on child OWO}

We found a significant combined effect of maternal $\mathrm{Hg}$ levels and either or both pre-pregnancy OWO and diabetes on child OWO risk. Children of OWO mothers in the top quartile of $\mathrm{Hg}$ had an increased risk of OWO $(\mathrm{RR}=1.87 ; 95 \%$ CI 1.55-2.25) compared to those with non-OWO mothers in lower quartiles of $\mathrm{Hg}$ (Fig. 2, a). Similarly, the risk of OWO was significantly higher in children of diabetic mothers in the top quartile of $\mathrm{Hg}$ than those with non-diabetic mothers in lower quartiles of $\mathrm{Hg}(\mathrm{RR}=1.45 ; 95 \% \mathrm{CI} 1 \cdot 15-1.83)$. When the presence of maternal OWO and diabetes was categorized into three groups: none (without OWO and diabetes), any (either OWO or diabetes), and both (OWO and diabetes), the risk of OWO $(\mathrm{RR}=2.06,95 \% \mathrm{CI} 1.56-2.71)$ was highest in children born to mothers with both OWO and diabetes and in the top quartile of $\mathrm{Hg}$, compared with children of mothers without OWO and diabetes and in low quartiles of $\mathrm{Hg}$ (Fig. 2a). However, there was no evidence of an interaction between maternal $\mathrm{RBC}-\mathrm{Hg}$ and pre-pregnancy OWO or between maternal $\mathrm{Hg}$ and diabetes with respect to the risk of child OWO $(p>0.05)$. These association patterns were similar for preschool age children (Fig. 2b), school age children (c), and adolescents (d).

\section{Counteracting effects of maternal folate status against RBC-Hg}

Maternal plasma folate was inversely related with $\mathrm{Hg}$ concentration (Additional file 1: Figure S3). As shown in Table 3, the rate of child OWO was significantly lower in children whose mothers in the highest $\mathrm{Hg}$ quartile and with adequate maternal folate than children whose mothers with a low folate levels (43.6\% vs. $60.9 \%$; $R R=$ 0.66 ; $95 \%$ CI $0.51-0.85 ; p=0.001)$. Adequate maternal 
Table 1 The characteristics of the study population $(n=1442)$

\begin{tabular}{|c|c|c|c|c|c|}
\hline & \multicolumn{5}{|c|}{ Maternal RBC mercury level $(\mu \mathrm{g} / \mathrm{L})$ in quartiles } \\
\hline & $\begin{array}{l}\mathrm{Q} 1 \\
0.39-1.04\end{array}$ & $\begin{array}{l}\text { Q2 } \\
1 \cdot 02-2 \cdot 10\end{array}$ & $\begin{array}{l}\text { Q3 } \\
2 \cdot 12-3.68\end{array}$ & $\begin{array}{l}\text { Q4 } \\
3 \cdot 70-27 \cdot 8 \\
\end{array}$ & $P$ trend \\
\hline \multicolumn{6}{|l|}{ Maternal characteristics } \\
\hline$n$ & 360 & 361 & 359 & 362 & \\
\hline Age, years & $26 \cdot 8 \pm 6 \cdot 5$ & $28 \cdot 0 \pm 6 \cdot 0$ & $29 \cdot 1 \pm 6 \cdot 7$ & $30 \cdot 3 \pm 6 \cdot 3$ & $<0.001$ \\
\hline Race & & & & & $<0.001$ \\
\hline Black & $192(53 \cdot 3)$ & $225(62 \cdot 3)$ & $265(73 \cdot 8)$ & $285(78 \cdot 7)$ & \\
\hline Non-black & $168(46 \cdot 7)$ & $136(37 \cdot 7)$ & $94(26 \cdot 2)$ & $77(21 \cdot 3)$ & \\
\hline Education & & & & & 0.367 \\
\hline High school and less & $234(65 \cdot 0)$ & $243(67 \cdot 3)$ & $233(64.9)$ & $226(62 \cdot 4)$ & \\
\hline Beyond high school & $126(35 \cdot 0)$ & $118(32 \cdot 7)$ & $126(35 \cdot 1)$ & $136(37 \cdot 6)$ & \\
\hline Smoking & & & & & $<0.001$ \\
\hline Not smoker & $279(77.5)$ & $284(78 \cdot 7)$ & $305(85 \cdot 0)$ & $324(89.5)$ & \\
\hline Smoker & $81(22.5)$ & $77(21 \cdot 3)$ & $54(15.0)$ & $38(10.5)$ & \\
\hline Parity & & & & & 0.001 \\
\hline Nulliparous & $177(49 \cdot 2)$ & $150(41 \cdot 6)$ & $139(38.7)$ & $136(37 \cdot 6)$ & \\
\hline Multiparous & $183(50 \cdot 8)$ & $211(58.4)$ & $220(61 \cdot 3)$ & $226(62 \cdot 4)$ & \\
\hline Pre-pregnancy BMl, kg/m² & $26 \cdot 4 \pm 6 \cdot 8$ & $27 \cdot 2 \pm 7 \cdot 6$ & $27 \cdot 2 \pm 6 \cdot 7$ & $26 \cdot 5 \pm 5 \cdot 7$ & 0.769 \\
\hline Overweight or obesity & $176(48.9)$ & $185(51 \cdot 2)$ & $203(56 \cdot 6)$ & $197(54 \cdot 4)$ & 0.063 \\
\hline Diabetes & $44(12 \cdot 2)$ & $45(12.5)$ & $45(12.5)$ & $50(13.8)$ & 0.537 \\
\hline Hypertensive disorder & $45(12.5)$ & $50(13.9)$ & $61(17.0)$ & $60(16 \cdot 6)$ & 0.068 \\
\hline Fish intake (serving/week) & & & & & $<0.001$ \\
\hline 0 & $163(45 \cdot 3)$ & $80(22 \cdot 2)$ & $55(15 \cdot 3)$ & $35(9.7)$ & \\
\hline $1-2$ & $183(50 \cdot 8)$ & $253(70 \cdot 1)$ & $246(68.5)$ & $239(66 \cdot 0)$ & \\
\hline$\geq 3$ & $14(3.9)$ & $28(7 \cdot 7)$ & $58(16 \cdot 2)$ & $88(24 \cdot 3)$ & \\
\hline Plasma folate $(\mathrm{nmol} / \mathrm{L})^{*}$ & $34.6(32.5-36.8)$ & $30 \cdot 1(28 \cdot 3-32 \cdot 1)$ & $29.4(27.5-31 \cdot 3)$ & $30 \cdot 7(28 \cdot 8-32 \cdot 7)$ & $<0.001$ \\
\hline \multicolumn{6}{|l|}{ Child's characteristics } \\
\hline Age, years & $7 \cdot 6 \pm 2.9$ & $8 \cdot 0 \pm 3 \cdot 2$ & $8.6 \pm 3.3$ & $8.4 \pm 3.0$ & 0.001 \\
\hline Gender & & & & & 0.002 \\
\hline Boy & $196(54.4)$ & $190(52 \cdot 6)$ & $180(50 \cdot 1)$ & $156(43 \cdot 1)$ & \\
\hline Girl & $164(45 \cdot 6)$ & $171(47.4)$ & $179(49.9)$ & $206(56 \cdot 9)$ & \\
\hline Birthweight, g & $2982 \pm 773$ & $2955 \pm 776$ & $2972 \pm 790$ & $3015 \pm 818$ & 0.775 \\
\hline Gestational age, weeks & $38 \cdot 0 \pm 3 \cdot 2$ & $37.9 \pm 3.3$ & $37 \cdot 8 \pm 3 \cdot 2$ & $38 \cdot 1 \pm 3 \cdot 1$ & 0.998 \\
\hline Preterm birth & $88(24.4)$ & $90(24.9)$ & $85(23.7)$ & $85(23.5)$ & 0.681 \\
\hline Fetal growth & & & & & 0.176 \\
\hline AGA & $290(80 \cdot 6)$ & $286(79 \cdot 2)$ & $279(77.7)$ & $281(77 \cdot 6)$ & \\
\hline SGA & $38(10.5)$ & $37(10 \cdot 3)$ & $39(10.9)$ & $37(10 \cdot 2)$ & \\
\hline LGA & $32(8.9)$ & $38(10.5)$ & $41(11.4)$ & $44(12 \cdot 2)$ & \\
\hline Breastfeeding & & & & & 0.013 \\
\hline Formula only & $90(25.0)$ & $105(29 \cdot 1)$ & $88(24.5)$ & $65(17.9)$ & \\
\hline Breastfeed exclusively & $28(7 \cdot 8)$ & $31(8 \cdot 6)$ & $21(5 \cdot 9)$ & $39(10 \cdot 8)$ & \\
\hline Both & $242(67 \cdot 2)$ & $225(62 \cdot 3)$ & $250(69 \cdot 6)$ & $258(71 \cdot 3)$ & \\
\hline Overweight or obesity & $138(38 \cdot 3)$ & $154(42 \cdot 7)$ & $146(40 \cdot 7)$ & $174(48 \cdot 1)$ & 0.019 \\
\hline Number of BMI measures & $3 \cdot 8 \pm 2 \cdot 3$ & $4 \cdot 0 \pm 2 \cdot 5$ & $4 \cdot 1 \pm 2 \cdot 6$ & $4 \cdot 0 \pm 2 \cdot 4$ & $<0.001$ \\
\hline
\end{tabular}



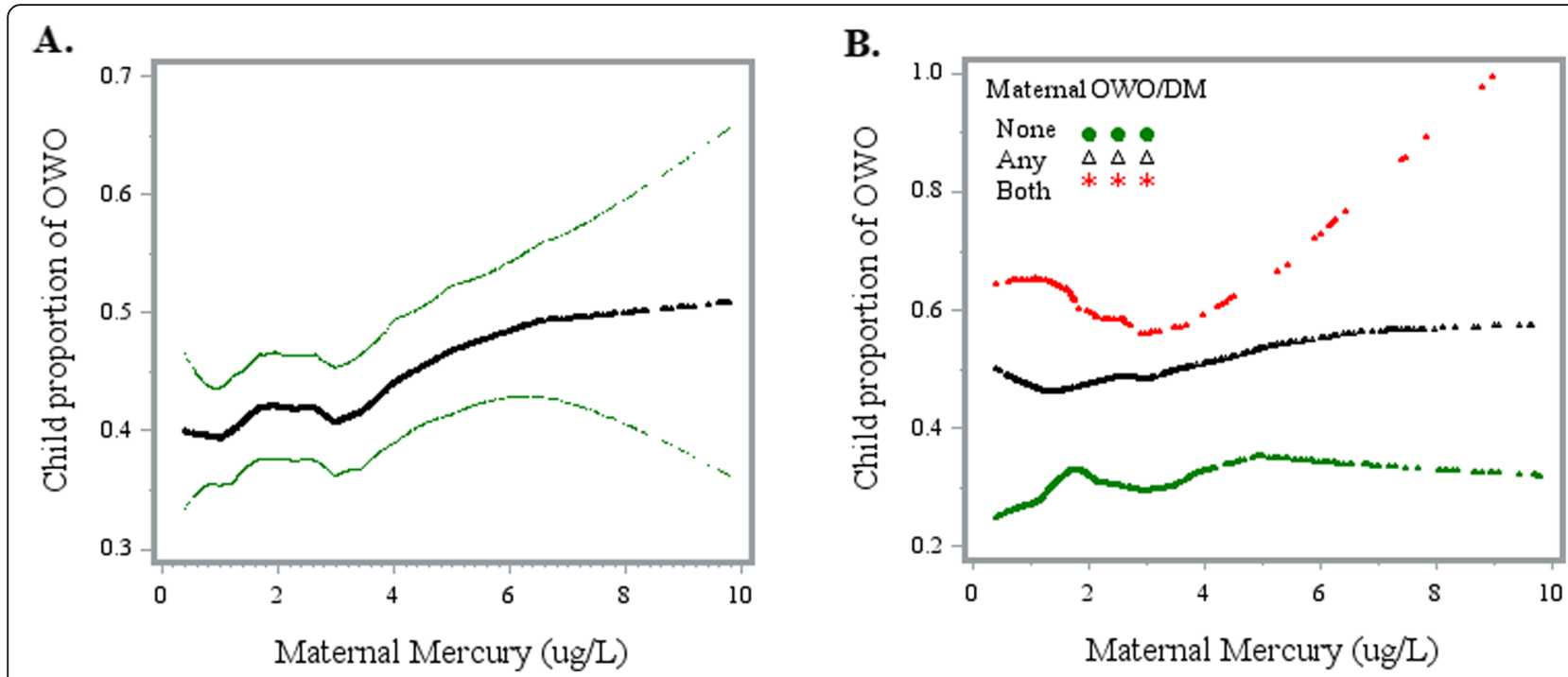

Fig. 1 Association between maternal RBC-Hg concentrations and offspring overweight or obesity. Abbreviation: OWO, overweight or obesity; DM, diabetes. Panel a displays the crude association between maternal RBC-Hg concentration and offspring proportion of OWO. Panel $\mathbf{b}$ displays the association stratified by maternal OWO/DM condition. None, non-OWO and non-diabetic; Any, either OWO or diabetes; Both, OWO and diabetes

folate status was not associated with reduced child OWO risk ( $\mathrm{RR}=0.96 ; 95 \%$ CI $0.80-1.15 ; p=0.658)$ among children of mothers with Hg levels in the lower three quartiles. The interaction between maternal RBC$\mathrm{Hg}$ and plasma folate on child OWO risk was marginal $(p=0.086$ for interaction).

\section{Subgroup and sensitivity analyses}

To control for other potential confounders, we further adjusted for frequency of maternal fish consumption (Additional file 1: Table S2), maternal RBC-selenium levels (Additional file 1: Table S3), and maternal RBC-lead levels (Additional file 1: Table S4). The results were similar to those reported above. A series of subgroup analyses were performed, and the associations were not changed materially among fish consumers (Additional file 1: Table S5), term births (Additional file 1: Table S6), Blacks (Additional file 1: Table S7), boys (Additional file 1: Table S8), girls (Additional file 1: Table S9), and breastfed children (Additional file 1: Table S10). To further evaluate the associations, we analyzed child BMI $z$ score as a continuous outcome (Additional file 1: Table S11), and again the results were consistent.

\section{Discussion}

To the best of our knowledge, this is the first study to investigate in utero $\mathrm{Hg}$ exposure and OWO in childhood using a prospective, longitudinal birth cohort. This study provided several new findings. Maternal RBC-Hg levels were positively associated with the risk of child OWO. The highest risk of child OWO was seen among children of mothers with $\mathrm{Hg}$ levels in the top quartile. The associations were consistent from preschool age to school age to adolescence. The Hg-related risk of child OWO was further enhanced by maternal pre-pregnancy OWO and/or diabetes but mitigated by adequate maternal folate status. These data suggest that in utero Hg exposure has a long-term consequence on child metabolic health, and optimal folate in utero may act to reduce $\mathrm{Hg}$ toxicity. Our findings underscore the need for screening for blood $\mathrm{Hg}$ and folate levels in pregnant women, particularly in OWO and/or diabetic women.

$\mathrm{Hg}$ is well-known to be a neurotoxicant and is beginning to be recognized as an obesogen $[6,12,13]$. Studies in adults have suggested that blood $\mathrm{Hg}$ levels were associated with obesity [6] and visceral adipose tissue (measured by dual-energy $X$-ray absorptiometry) [7]. Measures of $\mathrm{Hg}$ in human hair were also associated with BMI [13] and obesity [12]. Our study extends these findings, suggesting a positive association between maternal $\mathrm{Hg}$ levels and child risk of OWO from preschool age to school age to adolescence. These results were consistent across an array of sensitivity analyses. Our findings provide new evidence that in utero $\mathrm{Hg}$ exposure may play a role in the development of childhood OWO.

The underlying mechanism for the association between in utero $\mathrm{Hg}$ exposure and risk of child OWO is not clear. One potential mechanism is via $\mathrm{MeHg}$ induced oxidative stress and inflammation. Experimental studies have demonstrated that $\mathrm{MeHg}$ exposure induces oxidative stress and systemic inflammation [26], which, in turn, can cause disturbances in glucose metabolism and lipid peroxidation [27]. Consistently, a human study showed that $\mathrm{MeHg}$ exposure was associated with 
Table 2 Association of maternal mercury levels with child risk of overweight or obesity

\begin{tabular}{|c|c|c|c|c|c|c|c|c|c|}
\hline \multirow[t]{2}{*}{ Age group } & \multirow[t]{2}{*}{ Mercury quartile } & \multirow[b]{2}{*}{$n$} & \multirow[b]{2}{*}{ Case, $n(\%)$} & \multicolumn{3}{|c|}{ Crude } & \multicolumn{3}{|c|}{ Adjusted } \\
\hline & & & & $\mathrm{RR}$ & $95 \% \mathrm{Cl}$ & $p$ & $\mathrm{RR}$ & $95 \% \mathrm{Cl}$ & $p$ \\
\hline \multicolumn{10}{|c|}{ Total sample $2-15$ years $(n=1442)$} \\
\hline Q1 & & 360 & $138(38 \cdot 3)$ & 1.00 & & & 1.00 & & \\
\hline Q2 & & 361 & $154(42 \cdot 7)$ & $1 \cdot 11$ & $0.93-1 \cdot 33$ & 0.238 & 1.09 & $0.92-1 \cdot 30$ & 0.313 \\
\hline Q3 & & 359 & $146(40 \cdot 7)$ & 1.06 & $0.89-1 \cdot 27$ & 0.522 & 1.03 & $0.86-1.23$ & 0.748 \\
\hline Q4 & & 362 & $174(48 \cdot 1)$ & $1 \cdot 25$ & $1.06-1.49$ & 0.009 & $1 \cdot 24$ & $1.05-1.47$ & 0.013 \\
\hline$P$ trend & & & & & & 0.047 & & & 0.098 \\
\hline Q1-Q3 & & 1080 & $438(40 \cdot 6)$ & 1.00 & & & 1.00 & & \\
\hline Q4 & & 362 & $174(48 \cdot 1)$ & $1 \cdot 19$ & $1.04-1 \cdot 35$ & 0.010 & $1 \cdot 19$ & $1.05-1 \cdot 35$ & 0.007 \\
\hline \multicolumn{10}{|c|}{$2-5$ years $(n=1395)$} \\
\hline Q1 & & 346 & $113(32 \cdot 7)$ & 1.00 & & & 1.00 & & \\
\hline Q2 & & 347 & $139(40 \cdot 1)$ & 1.23 & $1 \cdot 01-1 \cdot 50$ & 0.044 & $1 \cdot 20$ & $0.99-1.45$ & 0.061 \\
\hline Q3 & & 347 & $138(39.8)$ & 1.22 & $1.00-1.49$ & 0.053 & $1 \cdot 15$ & $0.95-1.41$ & 0.160 \\
\hline Q4 & & 355 & $159(44 \cdot 8)$ & 1.37 & $1 \cdot 13-1 \cdot 66$ & 0.001 & $1 \cdot 33$ & $1 \cdot 10-1 \cdot 61$ & 0.003 \\
\hline$P$ trend & & & & & & 0.015 & & & 0.067 \\
\hline Q1-Q3 & & 1040 & $390(37.5)$ & 1.00 & & & 1.00 & & \\
\hline Q4 & & 355 & $159(44.8)$ & $1 \cdot 19$ & $1.04-1.37$ & 0.013 & $1 \cdot 19$ & $1.03-1.36$ & 0.016 \\
\hline \multicolumn{10}{|c|}{$6-9$ years $(n=1030)$} \\
\hline Q1 & & 250 & $100(40 \cdot 0)$ & 1.00 & & & 1.00 & & \\
\hline Q2 & & 255 & $118(46 \cdot 3)$ & $1 \cdot 16$ & $0.95-1.41$ & 0.156 & $1 \cdot 15$ & $0.94-1.39$ & 0.171 \\
\hline Q3 & & 258 & $116(45 \cdot 0)$ & $1 \cdot 12$ & $0.92-1.38$ & 0.259 & 1.09 & $0.89-1.34$ & 0.399 \\
\hline Q4 & & 267 & $142(53 \cdot 2)$ & 1.33 & $1 \cdot 10-1 \cdot 61$ & 0.003 & $1 \cdot 30$ & $1.07-1.58$ & 0.007 \\
\hline$P$ trend & & & & & & 0.028 & & & 0.100 \\
\hline Q1-Q3 & & 763 & $334(43 \cdot 8)$ & 1.00 & & & 1.00 & & \\
\hline Q4 & & 267 & $142(53 \cdot 2)$ & $1 \cdot 21$ & $1 \cdot 06-1 \cdot 40$ & 0.006 & $1 \cdot 20$ & $1 \cdot 04-1 \cdot 38$ & 0.010 \\
\hline \multicolumn{10}{|c|}{$10-15$ years $(n=449)$} \\
\hline Q1 & & 91 & $38(41 \cdot 8)$ & 1.00 & & & 1.00 & & \\
\hline Q2 & & 106 & $47(44 \cdot 3)$ & 1.06 & $0.77-1.47$ & 0.716 & $1 \cdot 14$ & $0.84-1.56$ & 0.383 \\
\hline Q3 & & 138 & $67(48 \cdot 6)$ & $1 \cdot 16$ & $0.86-1.57$ & 0.320 & 1.22 & $0.91-1.64$ & 0.184 \\
\hline Q4 & & 114 & $62(54 \cdot 4)$ & $1 \cdot 30$ & $0.97-1.75$ & 0.079 & $1 \cdot 40$ & $1.04-1 \cdot 88$ & 0.028 \\
\hline$P$ trend & & & & & & 0.057 & & & 0.058 \\
\hline Q1-Q3 & & 335 & $152(45 \cdot 4)$ & 1.00 & & & 1.00 & & \\
\hline Q4 & & 114 & $62(54.4)$ & $1 \cdot 20$ & $0.98-1.47$ & 0.083 & $1 \cdot 22$ & $0.99-1.50$ & 0.061 \\
\hline
\end{tabular}

Q quartile, $R R$ relative risk, Cl conference interval. Mercury quartile range: Q1 0.39-1.04 $\mu \mathrm{g} / \mathrm{L} ; \mathrm{Q} 21.04-2.10 \mu \mathrm{g} / \mathrm{L} ; \mathrm{Q} 32.12-3.68 \mu \mathrm{g} / \mathrm{L} ; \mathrm{Q} 43.70-27.8 \mu \mathrm{g} / \mathrm{L}$.

Adjusted for maternal age, race, smoking, education, parity, pre-pregnancy overweight or obesity, diabetes, hypertensive disorder, preterm birth, fetal growth pattern, and breastfeeding status

reduced activity of Paraoxonase 1 (PON1) [28], an enzyme that inhibits systemic oxidative stress and guards against atherosclerosis and obesity [29].

Nutrition has been proposed as a safe, simple, and inexpensive method to mitigate the detrimental effects of exposure to environmental toxicants [30, 31]. One of the nutrients that might reduce the adverse impacts of chemicals on the fetus is folate [18]. However, the literature is limited on the relationship between folate status and $\mathrm{Hg}$ toxicity. Previous studies showed that folate and vitamin B12 deficiency magnify the adverse effects of $\mathrm{Hg}$ [32] and that folate may play a role in alleviating $\mathrm{Hg}$ toxicity [19]. Our findings reiterate the potential importance of optimal maternal folate status in the setting of $\mathrm{Hg}$ exposure.

Our study has important public health implications. $\mathrm{MeHg}$ is a major contaminant in some fish and seafoods. The overall health impact of fish consumption may reflect both the beneficial effects of nutrients, such as omega-3 long-chain fatty acids, and the detrimental 


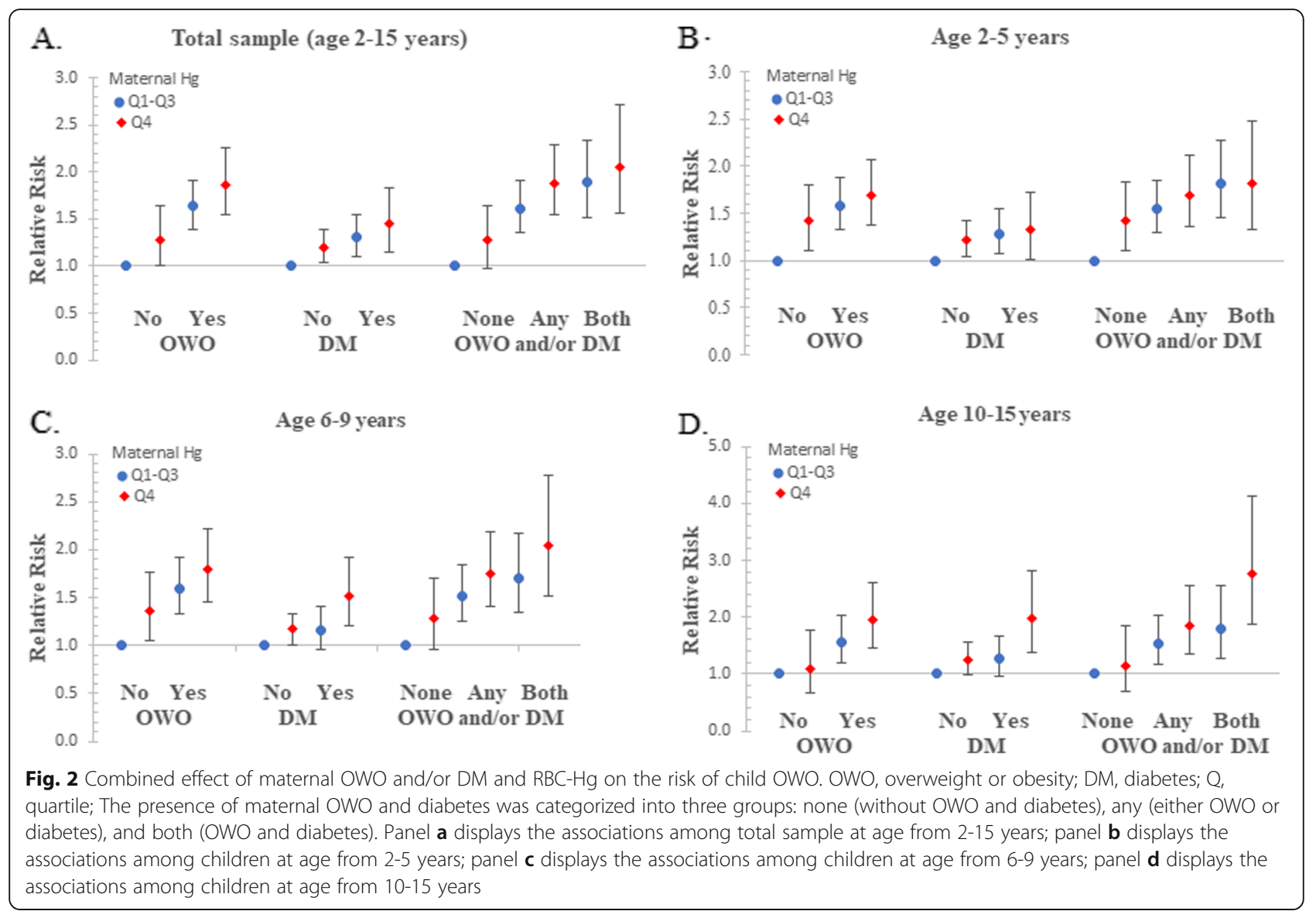

effects of contaminants, such as $\mathrm{Hg}$, found in fish. The results of the current study emphasize the need to carefully weigh the nutritional benefits of fish consumption with the risks of increased exposure to $\mathrm{Hg}$ during pregnancy to reduce the risk of childhood OWO. Our study findings raise the prospect to screen for $\mathrm{Hg}$ and folate and to optimize maternal folate status during gestation, especially among mothers with OWO and/or diabetes.

Our study has a couple of strengths. We measured child BMI longitudinally, which enabled us to define OWO over 15 years of follow-up and explore temporal characteristics of the association between in utero $\mathrm{Hg}$ exposure and OWO risk. In addition, we assessed in utero $\mathrm{Hg}$ exposure in maternal RBCs $1-3$ days after delivery, reflecting third trimester exposure and free from hemodilution. However, several potential limitations of the study need to be noted. We measured total $\mathrm{Hg}$, not $\mathrm{MeHg}$, though $\mathrm{Hg}$ concentrations in RBCs are the best biomarker of $\mathrm{MeHg}$ exposure insofar as 80\% of $\mathrm{MeHg}$ is stored in red blood cells [2]. Second, we did not measure child $\mathrm{Hg}$ exposure during childhood. Third, although we controlled for many potential confounders, such as maternal age, education, race/ethnicity, parity, smoking, and other pertinent covariates in the models, we could

Table 3 Modifying effects of maternal folate levels with mercury levels on child overweight or obesity risk

\begin{tabular}{|c|c|c|c|c|c|c|c|c|c|}
\hline \multirow[t]{2}{*}{ Mercury quantile } & \multirow[t]{2}{*}{ Maternal folate } & \multirow[b]{2}{*}{$n$} & \multirow[b]{2}{*}{ Case, $n(\%)$} & \multicolumn{3}{|c|}{ Crude } & \multicolumn{3}{|c|}{ Adjusted } \\
\hline & & & & $\overline{\mathrm{RR}}$ & $95 \% \mathrm{Cl}$ & $p$ & $\overline{\mathrm{RR}}$ & $95 \% \mathrm{Cl}$ & $p$ \\
\hline \multirow[t]{2}{*}{$\overline{\mathrm{Q} 4}$} & Low & 64 & 39 (60.9) & 1.00 & & & 1.00 & & \\
\hline & Adequate & 236 & $103(43.6)$ & 0.72 & $0.56-0.91$ & 0.007 & 0.66 & $0.51-0.85$ & 0.001 \\
\hline \multirow[t]{2}{*}{ Q1-Q3 } & Low & 195 & 85 (43.6) & 1.00 & & & 1.00 & & \\
\hline & Adequate & 714 & $289(40.5)$ & 0.93 & $0.77-1.11$ & 0.427 & 0.96 & $0.80-1.15$ & 0.658 \\
\hline$P$ for interaction & & & & & & 0.095 & & & 0.086 \\
\hline
\end{tabular}

Q quartile. Mercury quartile range: Q1 0.39-3.68 $\mu \mathrm{g} / \mathrm{L} ; \mathrm{Q} 43.70-27.8 \mu \mathrm{g} / \mathrm{L}$. Adequate folate was defined as plasma folate $\geq 20.4 \mathrm{nmol} / \mathrm{L}$; low folate was defined as plasma folate $<20.4 \mathrm{nmol} / \mathrm{L}$

Adjusted for maternal race, smoking, education, parity, pre-pregnancy overweight or obesity, diabetes, hypertensive disorder, preterm birth, fetal growth pattern, and breastfeeding status 
not completely eliminate the potential for residual confounding due to unknown or uncontrolled confounders. Finally, the fact that our study population was dominantly urban, low-income Blacks might limit the generalizability of our findings.

\section{Conclusion}

In this large, long-term prospective birth cohort study of a US urban low-income population, there was a significant dose-response relationship between in utero $\mathrm{Hg}$ exposure and risk of child OWO. Maternal pre-pregnancy OWO and/or diabetes enhanced the $\mathrm{Hg}$-child OWO associations, while adequate maternal folate status mitigated the associations. These findings, if further confirmed, underscore the need to screen for $\mathrm{Hg}$ and folate and to optimize maternal folate status during gestation, especially among mothers with OWO and diabetes.

\section{Supplementary information}

Supplementary information accompanies this paper at https://doi.org/10. 1186/s12916-019-1442-2.

Additional file 1: Figure S1. Flowchart of study population. Figure S2. The distribution of maternal RBC-mercury stratified by maternal race and prepregnancy overweight or obesity (OWO) and/or diabetes (DM) status. Figure S3. The relationship between maternal plasma folate and RBC-Hg concentrations. Table S1. Comparison of pre- and peri-natal characteristics between total enrolled sample, follow-up sample, included sample of this analysis and subset with folate data. Table $\mathbf{S 2}$. Individual and combined effects of maternal OWO and/or DM and mercury and child risk of OWO, with additional adjustment for frequency of fish consumption. Table S3. Individual and combined effects of maternal OWO and/or DM and mercury on child risk of OWO, with additional adjustment for maternal selenium level. Table S4. Individual and combined effects of maternal OWO and/or DM and mercury on child risk of OWO, with additional adjustment for maternal lead level. Table S5. Individual and combined effects of maternal OWO and/or DM and mercury on child OWO among fish consumers only $(n=1109)$. Table S6. Individual and combined effects of maternal OWO and/ or DM and mercury on child OWO among term births only $(n=1094)$. Table S7. Individual and combined effects of maternal OWO and/or DM and mercury on child OWO among black children $(n=967)$. Table s8. Individual and combined effects of maternal OWO and/or DM and mercury on child OWO among boys ( $n=722)$. Table S9. Individual and combined effects of maternal OWO and/or DM and mercury on child OWO among girls ( $n=720)$. Table S10. Individual and combined effects of maternal OWO and/or DM and mercury on child OWO among breastfed children only ( $n=1094)$. Table S11. Individual and combined effects of maternal OWO and/or DM and mercury on child BMl z-scores.

\section{Abbreviations}

AGA: Appropriate for gestational age; BBC: The Boston Birth Cohort; BMI: Body mass index; Cl: Confidence interval; CV: Coefficient variation; HELLP: Hemolysis, elevated liver enzymes, and low platelets syndrome; Hg: Mercury; IQR: Interquartile range; LGA: Large for gestational age; LOD: The limit of detection; MeHg: Methylmercury; OWO: Overweight or obesity; RR: Relative risk; SD: Standard deviation; SGA: Small for gestational age

\section{Acknowledgements}

The authors wish to acknowledge Thomas Kirn, PhD, Tina Fan, PhD, and Doug Haltmeier, MS in the New Jersey Department of Health for their leadership, input, and support throughout this project. The authors also wish to thank the study participants, the nursing staff at Labor and Delivery of the Boston Medical Center and the field team for their contributions to the
Boston Birth Cohort. Linda Rosen, MSEE, and the Clinical Data Warehouse assisted in obtaining relevant clinical information. She was compensated for her time. Clinical Data Warehouse service is supported by Boston University's Clinical and Translational Institute and the National Institutes of Health Clinical and Translational Science Award (grant U54-TR001012).

\section{Role of the funder/sponsor}

The funders had no role in the design and conduct of the study; collection, management, analysis, and interpretation of the data; preparation, review, or approval of the manuscript; and decision to submit the manuscript for publication. This information or content and conclusions are those of the authors and should not be construed as the official position or policy of, nor should any endorsements be inferred by the National Institutes of Health, the Health Resources and Services Administration, the US Department of Health and Human Services, or the US government, or the New Jersey Department of Health.

\section{Authors' contributions}

GW, JD, EB, and XW were responsible for study concept and design. GW, JD, and TRB were responsible for drafting of the manuscript. EB, AMS, and JM performed laboratory assays and quality control. GW, JD, DCB, MCW, XH, MWK, and TLC assumed major responsibilities for statistical analyses and data presentations. GW and YJ prepared blood samples for lab assay. XW was responsible for acquisition of the epidemiological and clinical data as well as biospecimen. All the authors (GW, JD, EB, AMS, JM, TRB, DCB, XH, YJ, MCW, TLC, MWK, XW) were responsible for critical review and revision of the manuscript and contributed to data interpretations. GW and XW have full access to all the data in the study and take responsibility for the integrity of the data and the accuracy of the data analysis. All authors read and approved the final manuscript.

\section{Funding}

The Boston Birth Cohort (the parent study) is supported in part by the National Institutes of Health (NIH) under grants number R01HD086013 and 2R01HD041702, and by the Health Resources and Services Administration of the U.S. Department of Health and Human Services under UJ2MC31074 Autism Longitudinal Data Project. Dr. Guoying Wang receives grant R03ES029594 from the NIH/National Institute of Environmental Health Science.

\section{Availability of data and materials}

The datasets used and/or analyzed during the current study are available from the corresponding author on reasonable request.

\section{Ethics approval and consent to participate}

The study has been approved by the Institutional Review Boards of Boston Medical Center and Johns Hopkins Bloomberg School of Public Health. Written informed consent was obtained from each study child's biological mother and assent from the study child.

\section{Consent for publication}

Not applicable.

\section{Competing interests}

The authors declare that they have no competing interests.

\section{Author details}

'Department of Population, Family and Reproductive Health, Center on the Early Life Origins of Disease, Johns Hopkins Bloomberg School of Public Health, 615 N. Wolfe Street, Baltimore, MD 21205-2179, USA. ${ }^{2}$ Division of Research, Office of Epidemiology and Research, Maternal and Child Health Bureau, Health Resources and Services Administration, 5600 Fishers Ln, Rockville, MD 20852, USA. ${ }^{3}$ Metals Laboratory, Environmental and Chemical Laboratory Services, The New Jersey Department of Health, Trenton, NJ 08628, USA. ${ }^{4}$ Mary Ann \& J. Milburn Smith Child Health Research, Outreach and Advocacy Center, Stanley Manne Children's Research Institute, Ann \& Robert H Lurie Children's Hospital of Chicago, 2430 N Halsted St, Chicago, IL 60614, USA. ${ }^{5}$ Department of Neurology, Boston Children's Hospital, Harvard Medical School, 300 Longwood Ave, Boston, MA 02115, USA. 'Department of Biostatistics, Johns Hopkins Bloomberg School of Public Health, 615 N. Wolfe street, Baltimore, MD 21205, USA. "Department of Environmental Health and Engineering, Johns Hopkins Bloomberg School of Public Health, 615 N. Wolfe street, Baltimore, MD 21205, USA. ${ }^{8}$ Department of Pediatrics, Johns Hopkins School of Medicine, $615 \mathrm{~N}$. Wolfe street, Baltimore, MD 21205, USA. 
Received: 11 June 2019 Accepted: 7 October 2019

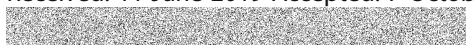

\section{References}

1. Shim YK, Lewin MD, Ruiz P, Eichner JE, Mumtaz MM. Prevalence and associated demographic characteristics of exposure to multiple metals and their species in human populations: the United States NHANES, 20072012. J Toxicol Environ Health A. 2017;80(9):502-12.

2. Clarkson TW, Magos L. The toxicology of mercury and its chemical compounds. Crit Rev Toxicol. 2006;36(8):609-62.

3. Grandjean P, Cordier S, Kjellstrom T, Weihe P, Budtz-Jorgensen E. Health effects and risk assessments. In: Pirrone N, Mahaffey KR, editors. Dynamics of mercury pollution on regional and global scales: atmospheric processes and human exposure around the world. Norwell: Springer; 2005. p. 499-523.

4. Guallar E, Sanz-Gallardo Ml, van't Veer P, Bode P, Aro A, Gomez-Aracena J, Kark JD, Riemersma RA, Martin-Moreno JM, Kok FJ, et al. Mercury, fish oils, and the risk of myocardial infarction. N Engl J Med. 2002;347(22):1747-54.

5. He K, Xun P, Liu K, Morris S, Reis J, Guallar E. Mercury exposure in young adulthood and incidence of diabetes later in life: the CARDIA trace element study. Diabetes Care. 2013;36(6):1584-9.

6. Cho S, Jacobs DR Jr, Park K. Population correlates of circulating mercury levels in Korean adults: the Korea National Health and Nutrition Examination Survey IV. BMC Public Health. 2014;14:527.

7. Park JS, Ha KH, He K, Kim DJ. Association between blood mercury level and visceral adiposity in adults. Diabetes Metab J. 2017;41(2):113-20.

8. Mone SM, Gillman MW, Miller TL, Herman EH, Lipshultz SE. Effects of environmental exposures on the cardiovascular system: prenatal period through adolescence. Pediatrics. 2004;113(4 Suppl):1058-69.

9. De Long NE, Holloway AC. Early-life chemical exposures and risk of metabolic syndrome. Diabetes Metab Syndr Obes. 2017;10:101-9.

10. Thayer KA, Heindel JJ, Bucher JR, Gallo MA. Role of environmental chemicals in diabetes and obesity: a National Toxicology Program workshop review. Environ Health Perspect. 2012;120(6):779-89.

11. Wang G, Hu FB, Mistry KB, Zhang C, Ren F, Huo Y, Paige D, Bartell T, Hong $X$, Caruso D, et al. Association between maternal prepregnancy body mass index and plasma Folate concentrations with child metabolic health. JAMA Pediatr. 2016:170(8):e160845.

12. Skalnaya MG, Demidov VA. Hair trace element contents in women with obesity and type 2 diabetes. J Trace Elem Med Biol. 2007;21(Suppl 1):59-61.

13. Skalnaya MG, Tinkov AA, Demidov VA, Serebryansky EP, Nikonorov AA, Skalny AV. Hair toxic element content in adult men and women in relation to body mass index. Biol Trace Elem Res. 2014;161(1):13-9.

14. Quig D. Cysteine metabolism and metal toxicity. Altern Med Rev. 1998;3(4): 262-70.

15. Zhao M, Chen YH, Dong XT, Zhou J, Chen X, Wang H, Wu SX, Xia MZ, Zhang C, Xu DX. Folic acid protects against lipopolysaccharide-induced preterm delivery and intrauterine growth restriction through its antiinflammatory effect in mice. PLoS One. 2013;8(12):e82713.

16. Joshi R, Adhikari S, Patro BS, Chattopadhyay S, Mukherjee T. Free radical scavenging behavior of folic acid: evidence for possible antioxidant activity. Free Radic Biol Med. 2001;30(12):1390-9.

17. Verhaar MC, Stroes E, Rabelink TJ. Folates and cardiovascular disease. Arterioscler Thromb Vasc Biol. 2002;22(1):6-13.

18. Ouyang F, Longnecker MP, Venners SA, Johnson S, Korrick S, Zhang J, Xu X, Christian P, Wang MC, Wang X. Preconception serum 1,1,1-trichloro2,2,bis(p-chlorophenyl)ethane and B-vitamin status: independent and joint effects on women's reproductive outcomes. Am J Clin Nutr. 2014;100(6): 1470-8.

19. Kim H, Kim KN, Hwang JY, Ha EH, Park H, Ha M, Kim Y, Hong YC, Chang N Relation between serum folate status and blood mercury concentrations in pregnant women. Nutrition. 2013;29(3):514-8.

20. Wang H, Mueller NT, Li J, Sun N, Huo Y, Ren F, Wang X. Association of maternal plasma folate and cardiometabolic risk factors in pregnancy with elevated blood pressure of offspring in childhood. Am J Hypertens. 2017; 30(5):532-40.

21. Wang G, Divall S, Radovick S, Paige D, Ning Y, Chen Z, Ji Y, Hong X, Walker SO, Caruso D, et al. Preterm birth and random plasma insulin levels at birth and in early childhood. Jama. 2014;311(6):587-96.

22. Huo Y, Li J, Qin X, Huang Y, Wang X, Gottesman RF, Tang G, Wang B, Chen $D, H e M$, et al. Efficacy of folic acid therapy in primary prevention of stroke among adults with hypertension in China: the CSPPT randomized clinical trial. Jama. 2015:313(13):1325-35.

23. Hong X, Wang G, Liu X, Kumar R, Tsai HJ, Arguelles L, Hao K, Pearson C, Ortiz K, Bonzagni A, et al. Gene polymorphisms, breast-feeding, and development of food sensitization in early childhood. J Allergy Clin Immunol. 2011;128(2):374-81 e372.

24. National Center for Health Statistics. CDC growth charts, United States. 2000 http://www.cdc.gov/growthcharts/. Accessed 26 Nov 2013.

25. National Center for Health Statistics. Overweight and obesity, defining childhood obesity. Page last reviewed: July 3, 2018. Accessed 30 July 2019

26. Yin Z, Milatovic D, Aschner JL, Syversen T, Rocha JB, Souza DO, Sidoryk M, Albrecht J, Aschner M. Methylmercury induces oxidative injury, alterations in permeability and glutamine transport in cultured astrocytes. Brain Res. 2007; 1131(1):1-10.

27. Chen YW, Huang CF, Tsai KS, Yang RS, Yen CC, Yang CY, Lin-Shiau SY, Liu $\mathrm{SH}$. The role of phosphoinositide 3-kinase/Akt signaling in low-dose mercury-induced mouse pancreatic beta-cell dysfunction in vitro and in vivo. Diabetes. 2006;55(6):1614-24.

28. Ayotte P, Carrier A, Ouellet N, Boiteau V, Abdous B, Sidi EA, Chateau-Degat $\mathrm{ML}$, Dewailly E. Relation between methylmercury exposure and plasma paraoxonase activity in inuit adults from Nunavik. Environ Health Perspect. 2011:119(8):1077-83.

29. Bhattacharyya T, Nicholls SJ, Topol EJ, Zhang R, Yang X, Schmitt D, Fu X, Shao M, Brennan DM, Ellis SG, et al. Relationship of paraoxonase 1 (PON1) gene polymorphisms and functional activity with systemic oxidative stress and cardiovascular risk. Jama. 2008;299(11):1265-76.

30. Furst A. Can nutrition affect chemical toxicity? Int J Toxicol. 2002;21(5):419-24.

31. Hennig B, Ettinger AS, Jandacek RJ, Koo S, McClain C, Seifried H, Silverstone A, Watkins B, Suk WA. Using nutrition for intervention and prevention against environmental chemical toxicity and associated diseases. Environ Health Perspect. 2007;115(4):493-5.

32. Hodgson NW, Waly MI, Al-Farsi YM, Al-Sharbati MM, Al-Farsi O, Ali A, Ouhtit A, Zang T, Zhou ZS, Deth RC. Decreased glutathione and elevated hair mercury levels are associated with nutritional deficiency-based autism in Oman. Exp Biol Med (Maywood). 2014;239(6):697-706.

\section{Publisher's Note}

Springer Nature remains neutral with regard to jurisdictional claims in published maps and institutional affiliations.

Ready to submit your research? Choose BMC and benefit from:

- fast, convenient online submission

- thorough peer review by experienced researchers in your field

- rapid publication on acceptance

- support for research data, including large and complex data types

- gold Open Access which fosters wider collaboration and increased citations

- maximum visibility for your research: over $100 \mathrm{M}$ website views per year

At BMC, research is always in progress.

Learn more biomedcentral.com/submissions 